\title{
Lipid hydrolysis products affect the composition of infant gut microbial communities in vitro
}

\author{
Rikke G. Nejrup ${ }^{1,2}$, Martin I. Bahl ${ }^{2}$, Louise K. Vigsnæs ${ }^{2}$, Christine Heerup ${ }^{1}$, Tine R. Licht ${ }^{2}$ and \\ Lars I. Hellgren ${ }^{1 *}$ \\ ${ }^{1}$ Center for Biological Sequence Analysis, Department of Systems Biology, Technical University of Denmark, Søltofts Plads, \\ Building 224, DK-2800 Kongens Lyngby, Denmark \\ ${ }^{2}$ National Food Institute, Technical University of Denmark, Mørkhøj Bygade 19, DK-2860 Søborg, Denmark
}

(Submitted 23 November 2014 - Final revision received 10 February 2015 - Accepted 18 February 2015 - First published online 20 May 2015)

\section{Abstract}

Some lipid hydrolysis products such as medium-chained NEFA (MC-NEFA), sphingosine and monoacylglycerols (MAG) possess antibacterial activity, while others, including oleic acid, are essential for the optimal growth of Lactobacillus species. Thus, changes in the concentrations of NEFA and MAG in the distal ileum and colon can potentially selectively modulate the composition of the gut microbiota, especially in early life when lipid absorption efficacy is reduced. As medium-chained fatty acids are enriched in mothers' milk, such effects may be highly relevant during gut colonisation. In the present study, we examined the effect of selected NEFA, MAG and sphingosine on the composition of faecal microbial communities derived from infants aged 2-5 months during a $24 \mathrm{~h}$ anaerobic in vitro fermentation. We tested lipid mixtures in the concentration range of $0-200 \mu \mathrm{M}$, either based on MC-NEFA (10:0 to 14:0 and MAG 12:0) or long-chained NEFA (LC-NEFA; 16:0 to 18:1 and MAG 16:0) with and without sphingosine, representing lipid hydrolysis products characteristic for intestinal hydrolysis of breast milk lipids. Ion Torrent sequencing of the bacterial $16 \mathrm{~S}$ ribosomal RNA gene revealed that the relative abundance of lactic acid-producing genera, including Lactobacillus and Bifidobacterium, was generally increased in the presence of $50 \mu \mathrm{m}$ or higher concentrations of MC-NEFA. For Bifidobacterium, the same effect was also observed in the presence of a mixture containing LC-NEFA with sphingosine. On the contrary, the relative abundance of Enterobacteriaceae was significantly decreased in the presence of both lipid mixtures. Our findings suggest that the high concentration of medium-chained fatty acids in breast milk might have functional effects on the establishment of the gut microbiota in early life.

Key words: Gut microbiota: Human milk lipids: Lipid hydrolysis products: $16 \mathrm{~S}$ ribosomal RNA sequencing

The important role of the human gut microbiota in relation to general health and well-being is now well established, and it is hence essential to obtain an understanding of the factors that form the composition of bacterial communities.

During birth and the first days of life, the gut microbiota starts to establish and evolve. The first colonisers play an important role, as the initial colonisation and community development is likely to affect the composition of the human microbiota throughout life ${ }^{(1)}$. Immediately after birth, bacteria originating from the diet and environment of the newborn child start colonising the gut. Some of these bacteria may originate directly from the breast milk $^{(2)}$. Facultative anaerobic bacteria are the first to establish in the gut ${ }^{(3,4)}$, generating an anaerobic environment, thus creating favourable conditions for obligate anaerobic bacteria ${ }^{(1,5)}$. In particular, establishment of lactic acid-producing bacteria, such as lactobacilli and bifidobacteria, have raised considerable interest. Besides their ability to enhance the bioavailability of nutrients, they have been shown to reduce symptoms associated with acute diarrhoea in infants ${ }^{(6)}$ as well as the occurrence of necrotising enterocolitis in preterm infants ${ }^{(7)}$. Rapidly, anaerobic bifidobacteria become the most dominating genus, typically represented mainly by the species $B$. longum ${ }^{(8)}$. The intestinal microbiota is relatively dynamic in early life, and its development depends on many different factors including diet, environment and host metabolic processes ${ }^{(9)}$.

The energy supply in early postnatal life originates mainly from lipids, as this is the main energy source in human breast milk ${ }^{(10,11)}$. The fatty acid (FA) composition of maternal milk is highly dependent on the mother's diet ${ }^{(12)}$; however,

Abbreviations: 16S rRNA, 16S ribosomal RNA; FA, fatty acids; LCFA, long-chained fatty acids; MAG, monoacylglycerols; MCFA, medium-chained fatty acids; PC, principal component; qPCR, quantitative PCR.

*Corresponding author: L. I. Hellgren, email lih@bio.dtu.dk 
the major FA are always a combination of medium-chained fatty acids (MCFA), such as caprylic acid (8:0), capric acid (10:0) and lauric acid (12:0) as well as the long-chained fatty acids (LCFA), such as myristic acid (14:0), palmitic acid (16:0), palmitoleic acid $(16: 1)$, stearic acid (18:0), oleic acid $(18: 1)$ and linoleic acid $(18: 2)^{(13-15)}$. In particular, the high concentration of MCFA is unique for milk. The total content of MCFA varies between 6 and $20 \mathrm{wt} \%$ of total $\mathrm{FA}^{(16)}$, with highest concentrations in situations with low maternal fat intake $^{(17)}$. The high concentration of MCFA is explained by the presence of a mammary-specific medium-chained acylthioester hydrolase that releases MCFA rather than palmitic acid, as the product of de novo lipogenesis ${ }^{(18)}$. To explain the presence of the, mammary-specific, synthesis of MCFA, it is assumed that they must fulfil important functions in neonate nutrition. In formula milk, lipids are most often added in the form of vegetable oils, thereby comprising primarily LCFA with no MCFA, if not specifically added. Another component found in human milk, not normally found in infant formulas, is sphingomyelin. Sphingomyelin typically constitutes 30$40 \%$ of phospholipids in breast milk ${ }^{(19,20)}$. In the gut, ingested sphingomyelin is first degraded to ceramide through the activity of the alkaline sphingomyelinase, and ceramide is further degraded to sphingosine by a neutral ceramidase, and finally sphingosine is absorbed by the enterocytes ${ }^{(21)}$.

Thus, the main differences in lipid hydrolysis products formed in the infant gut after feeding breast milk compared with infant formula are the presence of sphingosine and relatively high concentrations of MCFA. Interestingly, in studies on the skin microbiome, as well studies on lipid effects on foodborne pathogens, these two classes of molecules have been shown to possess potent anti-microbial activities, which may affect the composition of the skin microbiome ${ }^{(22-24)}$. Several studies have shown that sphingosine can have antimicrobial effects against a series of both Gram-negative and Gram-positive bacteria including Escherichia coli and Staphylococcus aureus $^{(24,25)}$. Furthermore, the most common MCFA in milk, lauric acid (12:0), also has antimicrobial effects against a number of bacteria including species of Fusobacterium, Clostridium, Salmonella and Listeria ${ }^{(22,24)}$. It is also noteworthy that the most common FA in human milk, oleic acid (18:1), has been found to stimulate the growth of several Lactobacillus species ${ }^{(26-29)}$. Thus, literature data suggest that lipid hydrolysis products characteristic for breast milk might be able to modulate community structure in the gut microbiota in different ways, compared with those formed after infant formula digestion.

For dietary lipids to have an impact on microbial communities, they must reach the part of the intestine highly colonised by the microbiota, i.e. the distal ileum and colon. The efficacy of lipid absorption varies; however, from studies of lipid residues in stool, it is clear that lipids are less well absorbed in infants than in adults ${ }^{(30)}$. Due to the low concentration of bile and the very low pancreatic lipase activity in the small intestine of newborns, TAG hydrolysis by the gastric lipase plays a major role in FA absorption in infants ${ }^{(10)}$. Gastric TAG hydrolysis is higher after consumption of breast milk than that of infant formula ${ }^{(31,32)}$; hence, FA absorption is lower from formula than breast milk, which also is reflected in a higher faecal fat excretion in infants fed with formula than breast milk ${ }^{(33)}$. The amount of fat excreted with the stool has been found to be up to $20-30 \%$ in preterm infants, $10 \%$ in term infants and less than $5 \%$ in adults ${ }^{(10)}$. Little is known about the concentrations of specific milk FA in the intestinal tract of human infants; however, caecal content of MCFA in piglets fed diets containing similar concentrations of MCFA as in human milk (approximately $3 \mathrm{wt} \%$ compared to approximately $6 \%$ in human milk) has been found to be about $200 \mu \mathrm{mol} / \mathrm{kg}^{(34)}$, indicating that the concentration of MCFA in the distal ileum is high enough to have an impact on bacterial growth. It is also well known that degradation of sphingomyelin is a slow process that continues through the entire gut including the colon ${ }^{(21)}$, thus the entire microbiota will be exposed to sphingosine released in this process. Despite the variation in lipid hydrolysis products that reach the bacteria-rich environments of the gut, very little is known about the influence of specific lipids on intestinal bacterial composition.

Breast-fed infants have a higher relative abundance of Bifidobacterium compared with formula-fed infants ${ }^{(35)}$, which is consistent with studies demonstrating that human milk oligosaccharides selectively stimulate bifidobacteria ${ }^{(36)}$. However, we hypothesise that lipid hydrolysis products from human milk fat may also influence the composition of the gut microbiota. Thus, the aim of the present study was to investigate how lipid hydrolysis products characteristic for the hydrolysis of human milk, rather than infant formula, affect the composition of microbial communities originating from infants during a $24 \mathrm{~h}$ in vitro fermentation.

\section{Materials and methods}

\section{Experimental set-up}

Sample collection and processing. Faecal samples from nine healthy infants (aged 2-5 months) were collected as inoculum for in vitro fermentation. Of these infants, one was born by caesarean section, and at the time of sampling, all infants were breast-fed. Neither the infants nor their mothers were treated with antibiotics during the last 3 months before sample collection. Faecal samples were collected at the home of the participants, where diapers right after defecation were compressed in a tightly sealed plastic bag, minimising air exposure. Diapers were stored at $+5^{\circ} \mathrm{C}$ until further processing in the laboratory. Storage time was limited to a maximum of $3 \mathrm{~h}$. Faecal samples were prepared for in vitro fermentation in an anaerobic cabinet $\left(10 \% \mathrm{H}_{2}, 10 \% \mathrm{CO}_{2}\right.$ and $80 \% \mathrm{~N}_{2}$; Macs Work Station, Don Whitley Scientific Limited). Faecal samples were taken from the middle of each stool and homogenised in $50 \%$ pre-reduced glycerol in the ratio of $1: 1$. The samples were stored at $-80^{\circ} \mathrm{C}$ until use.

Informed consent for the use of the children's samples in the study was obtained from all mothers.

Small-scale in vitro fermentation. To examine the effect of lipid hydrolysis products on microbial composition in infant faecal samples, a small-scale $(4 \mathrm{ml})$ batch in vitro fermentation 
method was used. To a fermentation medium rich in carbohydrates, mixtures of NEFA, monoacylglycerols (MAG) and sphingosine were added in various physiological relevant concentrations, as described below, mimicking the composition of lipid hydrolysis products from TAG in human milk fat.

Preparation of fermentation medium. The fermentation medium contained (per litre) ${ }^{(37)}: 2 \cdot 0 \mathrm{~g}$ peptone water (Oxoid Limited); $3.0 \mathrm{~g}$ yeast extract (normally $1.0 \mathrm{~g}$; Oxoid Limited); $0.1 \mathrm{~g} \mathrm{NaCl}$ (Merck KGaA); $0.04 \mathrm{~g} \mathrm{KH}_{2} \mathrm{PO}_{4}$ (Merck KGaA); $0.01 \mathrm{~g} \quad \mathrm{MgSO}_{4} .7 \mathrm{H}_{2} \mathrm{O} \quad$ (Merck KGaA); $0.01 \mathrm{~g} \quad \mathrm{CaCl}_{2} .2 \mathrm{H}_{2} \mathrm{O}$ (Sigma-Aldrich); $2 \mathrm{~g} \mathrm{NaHCO}_{3}$ (Sigma-Aldrich); $1.0 \mathrm{ml}$ Resazurin solution (0.05 g Resazurin (Sigma-Aldrich) in $100 \mathrm{ml}$ ion-exchanged water). To mimic the intestinal environment and concurrently not to limit the bacterial growth by the fermentation medium itself, the following ingredients were added (per litre): $4.0 \mathrm{~g}$ mucin from porcine stomach (SigmaAldrich); $1.0 \mathrm{~g}$ arabinogalactan (Sigma-Aldrich); $2.0 \mathrm{~g}$ pectin from apple (Sigma-Aldrich); $1.0 \mathrm{~g}$ xylan from beechwood (Sigma-Aldrich); $3.0 \mathrm{~g}$ starch from potatoes (Sigma-Aldrich); $0 \cdot 4 \mathrm{~g}$ glucose (Merck KGaA).

Finally, a mixture of FA emulsified in bile salt was added, as described below.

Preparation of fatty acid mixtures. Mixtures of FA, MAG and sphingosine were prepared in four different combinations, each mimicking a part of the lipid hydrolysis product from human milk fat. Mixture I (MCFA) comprising capric acid (10:0), lauric acid (12:0) and myristic acid (14:0) were mixed in the ratio 10:65:25. Lauric acid as 2-MAG was added, giving an overall NEFA:MAG ratio of 2:1. Mixture II (MCFA + 10 mol $\%$ sphingosine) consisted of mixture I + additional $10 \mathrm{~mol} \%$ sphingosine. Mixture III (LCFA) comprising palmitic acid (16:0), stearic acid (18:0) and oleic acid (18:1) were mixed in a ratio of 50:10:40. Palmitic acid as 2-MAG was added, giving an overall NEFA:MAG ratio of 2:1. Mixture IV (long-chained NEFA $+10 \mathrm{~mol} \%$ sphingosine) comprised mixture III + additional $10 \mathrm{~mol} \%$ sphingosine.

All lipids were from Sigma-Aldrich, except for lauric acid as 2-MAG being from Larodan AB.

The lipid mixtures were prepared in chloroform-methanol (2:1), and the organic solvent was evaporated under $\mathrm{N}_{2}$. The lipid mixtures were emulsified by the addition of $0.5 \mathrm{~g} / \mathrm{l}$ porcine bile salt (Sigma-Aldrich) mixture dissolved in ionexchanged water, and sonicated (Kem-En-Tec Nordic A/S) for $3 \mathrm{~min}$.

The lipid mixtures were added to the fermentation medium to a final concentration of $0,10,50,100$ and $200 \mu \mathrm{m}$ of combined NEFA and 2-MAG. In mixtures II and IV, additionally $10 \mathrm{~mol} \%$ sphingosine was added corresponding to final concentrations of $0,1,5,10$ and $20 \mu \mathrm{m}$.

After lipid addition, $\mathrm{pH}$ was adjusted to $7 \cdot 0$ using $1 \mathrm{M}-\mathrm{HCl}$ followed by autoclaving at $121^{\circ} \mathrm{C}$ for $15 \mathrm{~min}$.

After autoclaving, the following compounds were added (per litre): $0.5 \mathrm{~g} \mathrm{L-cysteine-HCl}$ solution ( $100 \mathrm{~g} / \mathrm{l}$ L-cysteine$\mathrm{HCl}$ (Sigma-Aldrich) in ion-exchanged water, filtered); $10 \mathrm{ml}$ Hemin solution $(50 \mathrm{mg}$ Hemin (Sigma-Aldrich) in $1.0 \mathrm{ml}$ of $1 \mathrm{M}-\mathrm{NaOH}$ and $100 \mathrm{ml}$ ion-exchanged water, filtered); $2.0 \mathrm{ml}$ vitamin $\mathrm{K}_{1}$ solution $\left(0 \cdot 15 \mathrm{ml}\right.$ vitamin $\mathrm{K}_{1}$ (Sigma-Aldrich) in $30 \mathrm{ml}$ ethanol (96\%; CCS Healthcare AB)).
The autoclaved fermentation medium was pre-reduced overnight at $+37^{\circ} \mathrm{C}$ in an anaerobic cabinet before use.

Anaerobic in vitro fermentation. Faecal samples (1:1 in glycerol) were defrosted and diluted at 1:5 in pre-reduced PBS ( $\mathrm{pH} 7$ ). The samples were hereafter further diluted in the fermentation medium (1:10), resulting in a final dilution of 1:100. In vitro fermentation was conducted in an anaerobic cabinet for $24 \mathrm{~h}$, and all fermentation experiments were performed in triplicate. During fermentation, tubes were slightly shaken and caps were loosely tightened to allow gas exchange, with little evaporation. The samples $(4 \mathrm{ml})$ for analysis of changes in the composition of bacterial communities were obtained at the beginning of fermentation $\left(t_{0}\right)$ and after $24 \mathrm{~h}\left(t_{24}\right)$, and $\mathrm{pH}$ was measured at both time points.

\section{Sample analysis}

\section{Bacterial distribution}

Purification of bacterial DNA. Total bacterial DNA from fermentation was extracted and purified using a QIAamp DNA stool mini kit (Qiagen), according to the manufacturer's instructions. In advance, a bead-beating step was included using $0 \cdot 1 \mathrm{~mm}$ zirconia-silica beads (Bio Spec Products, Inc.). DNA concentrations were measured using the Qubit ${ }^{\circledR}$ dsDNA HS Assay Kit (Thermo Fisher Scientific Inc.) with the Qubit ${ }^{\circledR} 2.0$ Fluorometer. Purified DNA was stored at $-20^{\circ} \mathrm{C}$ until use.

Real-time quantitative PCR assay conditions. Changes in faecal microbial communities were monitored at the two time points ( $t_{0}$ and $t_{24}$ ) by real-time quantitative PCR (qPCR) using selected validated primers (Table 1), with apparatus, running conditions and data processing procedure as described previously ${ }^{(38)}$.

Sequencing of $16 \mathrm{~S}$ ribosomal RNA encoding gene amplicons. For samples fermented with a lipid mixture concentration of 0 , 50 and $100 \mu \mathrm{m}$, respectively, $16 S$ ribosomal RNA (rRNA) gene sequencing was performed on the Ion Torrent platform, as described previously ${ }^{(39)}$. Briefly, the V3-region of the $16 \mathrm{~S}$ rRNA gene was amplified using a universal forward primer (PBU 5'-A-adapter-TCAG-barcode-CCTACGGGAGGCAGCAG-3') with a unique $10-12$ bp barcode for each bacterial community (IonXpress barcode as suggested by the supplier; Life Technologies) and an universal reverse primer (PBR $5^{\prime}$-trP1adapter-ATTACCGCGGCTGCTGG-3'). Sequencing was carried out on an Ion OneTouch ${ }^{\mathrm{TM}}$ platform (Ion Torrent ${ }^{\mathrm{TM}}$; Life Technology) using a 318-V2 chip. Sequence data were obtained in the FASTQ format and further processed using the CLC bio genomic workbench (Qiagen) in order to de-multiplex and remove sequencing primers and perform quality trimming.

Taxonomic assignment to $16 \mathrm{~S}$ reads and data handling. Ribosomal Database Project Classifier software (RDP 10 database, Update 18) was used to classify the sequences ${ }^{(40)}$. At the phylum, family and genus levels, the relative abundance of bacterial taxa was calculated as a percentage of the total number of reads for each sample. Fold differences were calculated for both 50 and $100 \mu \mathrm{m}$ samples compared with the control samples $(0 \mu \mathrm{M})$. 
Table 1. List of primer set targeting regions within the $16 S$ ribosomal RNA gene

\begin{tabular}{|c|c|c|c|c|c|}
\hline Phylum & Genus & Species & Primer sequence $\left(5^{\prime}-3^{\prime}\right)$ & $\begin{array}{l}\text { Amplicon } \\
\text { size }^{\star}(b p)\end{array}$ & References \\
\hline Universal HDA (V3) & All & All & $\begin{array}{l}\text { ACTCCTACGGGAGGCAGCAGT } \\
\text { GTATTACCGCGGCTGCTGGCAC }\end{array}$ & $174-199$ & Walter et al. ${ }^{(49)}$ \\
\hline Actinobacteria & Bifidobacterium & spp. & $\begin{array}{l}\text { CGCGTCYGGTGTGAAAG } \\
\text { CCCCACATCCAGCATCCA }\end{array}$ & 244 & Delroisse et al. ${ }^{(50)}$ \\
\hline Actinobacteria & Bifidobacterium & adolescentis & $\begin{array}{l}\text { CTCCAGTTGGATGCATGTC } \\
\text { CGAAGGCTTGCTCCCAGT }\end{array}$ & 279 & Matsuki et al. ${ }^{(51)}$ \\
\hline Actinobacteria & Bifidobacterium & breve & $\begin{array}{l}\text { AATGCCGGATGCTCCATCACAC } \\
\text { GCCTTGCTCCCTAACAAAAGAGG }\end{array}$ & 286 & Rinne et al. ${ }^{(52)}$ \\
\hline Actinobacteria & Bifidobacterium & $\begin{array}{l}\text { catenulatum/ } \\
\text { pseudocatenulatum }\end{array}$ & $\begin{array}{l}\text { CGGATGCTCCGACTCCT } \\
\text { CGAAGGCTTGCTCCCGAT }\end{array}$ & 289 & Matsuki et al. ${ }^{(51)}$ \\
\hline Actinobacteria & Bifidobacterium & longum & $\begin{array}{l}\text { GGATGTTCCAGTTGATCGCATGGTC } \\
\text { AGCCGGTGCTTATTCAACGGGTAA }\end{array}$ & 312 & Bergstrom et al. ${ }^{(38)}$ \\
\hline Firmicutes & Lactobacillus & spp. & AGCAGTAGGGAATCTTCCA & 341 & $\begin{array}{l}\text { Walter et al. }{ }^{(53)} \\
\quad \text { and Heilig et al. }{ }^{(54)}\end{array}$ \\
\hline Firmicutes & Lactobacillus & acidophilus & $\begin{array}{l}\text { CACCGCTACACATGGAG } \\
\text { GCAGATCGCATGATCAGCTTATA } \\
\text { TCAGTCTCTCAACTCGGCTATG }\end{array}$ & 129 & Firmesse et al. ${ }^{(55)}$ \\
\hline
\end{tabular}

*Approximate amplicon size.

SCFA analysis. Analysis of SCFA is a slightly modified version of a previously published method ${ }^{(41)}$. The method was validated through spiking of authentic samples with increasing concentrations of the standards used in the calibration curve before sample homogenisation and analyses, and determining intra- and inter-assay variability. Intra-assay variability was on average for the different SCFA determined to be $5.4 \%$ and average inter-assay variability to be $8.6 \%$.

For quantification, a combination of internal and external calibration was used. As an internal standard, $5 \mu \mathrm{mol}$ 2-ethyl butyric acid dissolved in 10\% formic acid was added to $500 \mu \mathrm{l}$ of each sample before sample preparation. Calibration curves of standard mixtures containing acetic, propionic, butyric, iso-butyric, iso-valeric, valeric, caproic, 2-ethyl butyric acids (Sigma-Aldrich) in concentrations ranging from $20 \mu \mathrm{M}$ to $20 \mathrm{~mm}$ were analysed in the same GC sequences as the samples on each day of the analyses. After addition of the internal standard, the fermentation fluid was centrifuged at $10000 \mathrm{~g}$ for $10 \mathrm{~min}$, the supernatant was filtered through $0.2 \mu \mathrm{m}$ Phenex-NY syringe filters (Phenomenex) and transferred to GC vials. The analysis was performed using a HP 6890 GC system from Agilent Technologies with a CP-FFA wall-coated open tubular (WCOT) fused silica capillary column $(25 \mathrm{~mm} \times 0.53 \mathrm{~mm} \times 1 \mu \mathrm{m}$ film thickness; Chrompack). The injector and detector temperature was $250^{\circ} \mathrm{C}$, and He gas was used as a carrier (constant flow rate $1 \mathrm{ml} / \mathrm{min}$ ). The initial temperature at $60^{\circ} \mathrm{C}$ was kept for $30 \mathrm{~s}$; thereafter, the temperature was raised to $180^{\circ} \mathrm{C}$ with $10^{\circ} \mathrm{C} / \mathrm{min}$. This temperature was kept for 3 min before it was increased with $20^{\circ} \mathrm{C} / \mathrm{min}$ to a final temperature at $215^{\circ} \mathrm{C}$, which was kept for $5 \mathrm{~min}$ before re-setting of the system to the initial temperature. The total GC programme lasted for $22 \mathrm{~min}$. Data collection and peak integration was performed using the OpenLAB Chromatography Data System (CDS) ChemStation Edition software. Peak integration was manually controlled and manually optimised when needed.
SCFA concentration in the samples was determined based on the external standard for each SCFA, and normalised to the concentration of the internal standard to compensate for losses during sample preparation.

\section{Statistical analysis}

Data are presented as box and whisker plots with 5th-95th percentiles. Statistical analysis was performed using the GraphPad Prism 5.00 software (GraphPad Software, Inc.) and the R software package version 3.0.2 (http://www.r-project.org).

The effect of lipid hydrolysis products on bacterial composition was analysed using a two-sample permutation test, followed by a calculation of false discovery rate indicated as a $q$ value. The $q$ values should be interpreted as the probability of obtaining false discoveries within the given test set of a total of 168 (phylum, family and genus levels). Differences between groups were considered statistically significant if $q$ values $<0.05$ were obtained. Correlations between groups and lipid concentration were calculated using a Pearson's test for normally distributed data and a Spearman's rank correlation test for non-parametric data. The distribution of each dataset was tested using a D'Agostino-Pearson omnibus K2 normality test with a significance level at $0 \cdot 05$.

Principal component (PC) analysis of the relative bacterial composition at the family level, based on the $16 \mathrm{~S}$ rRNA gene sequencing, was performed on auto-scaled data using the software package LatentiX $2 \cdot 11$ (Latent5; http://www. latentix.com). Score values for PC 1, 2 and 3 were calculated for the three tested concentrations $(0,50$ and $100 \mu \mathrm{M})$ of all the sequenced samples.

\section{Results}

\section{Bacterial distribution}

Bacterial composition of the inoculum. Sequencing of the infant faecal samples used as inoculum in the anaerobic 
in vitro fermentation resulted in the classification of more than $99 \%$ of the bacteria at the genus level, while the entire community was characterised at the phylum and family levels (Fig. 1). Individual ' $\mathrm{C}$ ' was delivered by caesarean section.

At the phylum level (Fig. 1(a)), the microbial communities were dominated by Actinobacteria in six of the donors and by Firmicutes in the remaining three. Together, these phyla accounted for more than $73 \%$ of the total bacterial population in all donors. Bacteroidetes constituted between 5 and $10 \%$ of the total population, except in two samples (A and H), which contained no Bacteroidetes. Proteobacteria accounted for no more than 5\%, except in two samples (A and $\mathrm{H}$ ), which contained 27 and $12 \%$ Proteobacteria, respectively.

At the family level (Fig. 1(b)), Actinobacteria were dominated by Bifidobacteriaceae and to a lower extent by Coriobacteriaceae. Additionally, Micrococcaceae and Actinomycetaceae were found in low levels $(<0.2 \%)$ in half of the samples.

The families within Firmicutes varied greatly between donors. The most common families were Enterococcaceae, Peptostreptococcaceae, Ruminococcaceae, Lachnospiraceae and Veillonellaceae. However, also Erysipelotrichaceae, Lactobacillaceae and Acidaminococcaceae were found in a few of the samples.

Within the Bacteroidetes, Bacteroidaceae was the most abundant family found in seven out of nine samples. Porphyromonadaceae were found in half of the samples with abundances up to $7 \%$. Likewise, Rikenellaceae were found in half of the donors, but in lower levels $(<1 \%)$. Enterobacteriaceae accounted for most of the families within Proteobacteria, constituting typically $3 \%$ of the population, except in two samples (A and H), which had levels of 27 and $12 \%$. Halomonadaceae, Desulfovibrionaceae and Sutterella were found in half of the donors in levels below $2 \%$.

At the genus level (results not shown), Bifidobacterium dominated Actinobacteria, whereas Streptococcus, Veillonella and Blautia dominated Firmicutes. Lactobacillaceae were represented by Lactobacillus and Bacteroides constituted most of the Bacteroidetes, while Enterobacteriaceae were either represented by Klebsiella or Escherichia/Shigella.

Effect of fermentation with lipids on lactic acid-producing bacteria as determined by quantitative PCR. qPCR revealed that fermentation with $50 \mu \mathrm{M}$ or higher concentrations of MCFA resulted in higher relative abundances of Bifidobacterium than observed in fermented controls with no added MCFA (50 $\mu \mathrm{M}: P=0.003, q=0.039 ; 100 \mu \mathrm{M}: P=0.004, q=0.029$ and $200 \mu \mathrm{M}$ : $P=0.003, q=0.029$; Fig. $2(\mathrm{a})$ ). The relative abundance of Bifidobacterium was significantly correlated with the concentration of MCFA added up to $100 \mu \mathrm{M}(P=0.035$, $R^{2}$ 0.9930; results not shown). This effect of MCFA was not present when $10 \mathrm{~mol} \%$ sphingosine was added to the MCFA mixture (Fig. 2(c)). Interestingly, while the addition of LCFA alone did not affect the growth of Bifidobacterium (Fig. 2(b)), a combination of LCFA and $10 \mathrm{~mol} \%$ sphingosine promoted their growth $(100 \mu \mathrm{M}: P=0.0111, \quad q=0.0329$; Fig. 2(d)).

The relative abundance of Lactobacillus was significantly higher after addition of MCFA at concentrations of 50 and

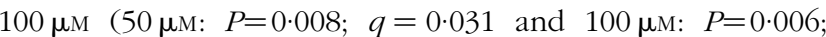
$q=0.031$; Fig. 2(e)); however, no correlation was observed between the relative abundance and concentration of MCFA. Neither MCFA in connection with sphingosine (Fig. 2(g)) nor any of the LCFA mixtures had an effect on the growth of Lactobacillus (Fig. 2(f) and (h)).

Additional qPCR was conducted using primers targeting specific species within Bifidobacterium and Lactobacillus. Here, B. longum was found in all individuals, and a stimulatory effect of MCFA on their growth was observed $(50 \mu \mathrm{M}$ : $P=0.028, q=0.040 ; 100 \mu \mathrm{M}: P=0.004, q=0.029$ and $200 \mu \mathrm{M}$ : $P=0.005, q=0.029$; Fig. 2(i)), as was also observed for the Bifidobacterium genus, this effect was dose-dependent up to $100 \mu \mathrm{M}$ ( $P=0 \cdot 010, R^{2}$ 0.9802; results not shown). A Pearson's (a)

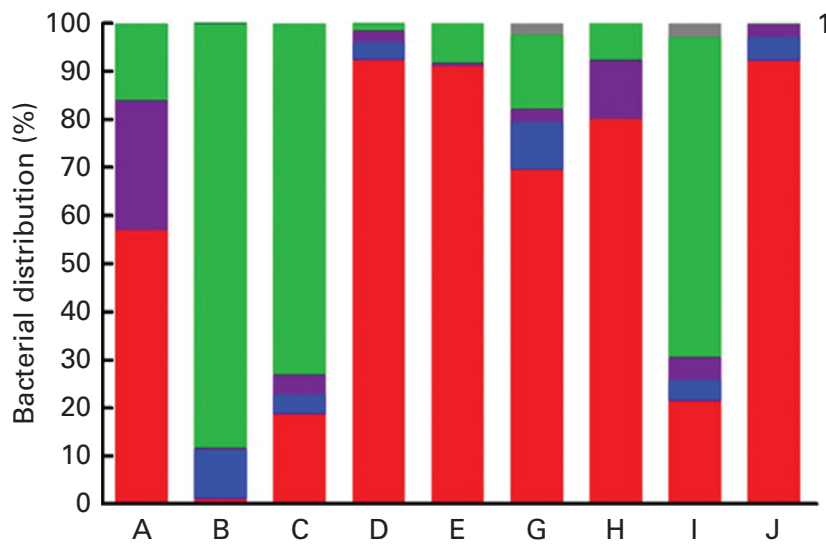

(b)

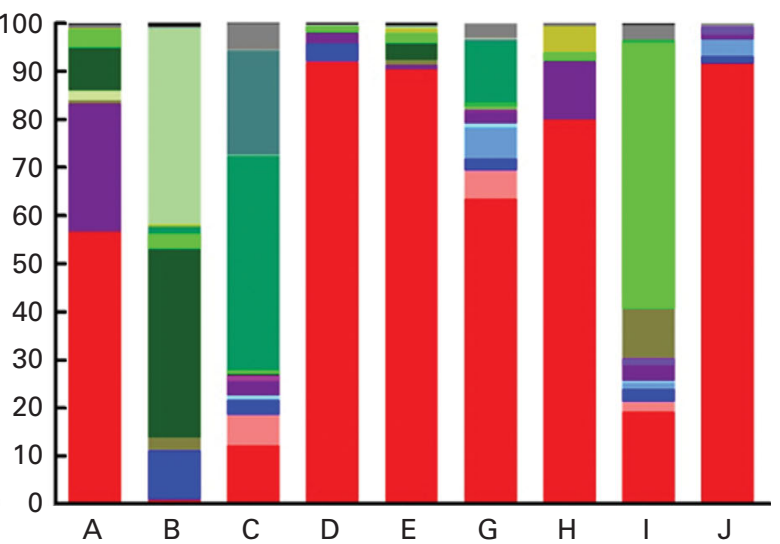

Fig. 1. Bacterial distribution (\%) in infant donor material used as inoculum in the anaerobic in vitro fermentation. Distributions are shown as a percentage at the phylum (a) and family (b) levels. The distribution at the genus level can be found in the text. Letters (A-J) indicate each individual. (a): $\square$, Actinobacteria; $\square$,

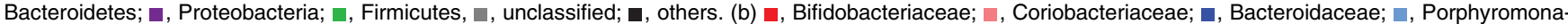

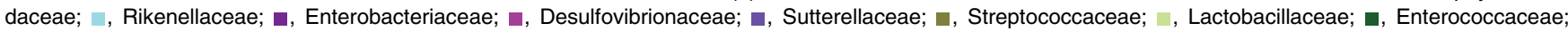

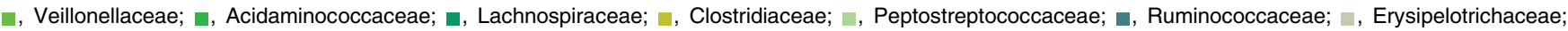
, unclassified; $\mathbf{\square}$, others. 

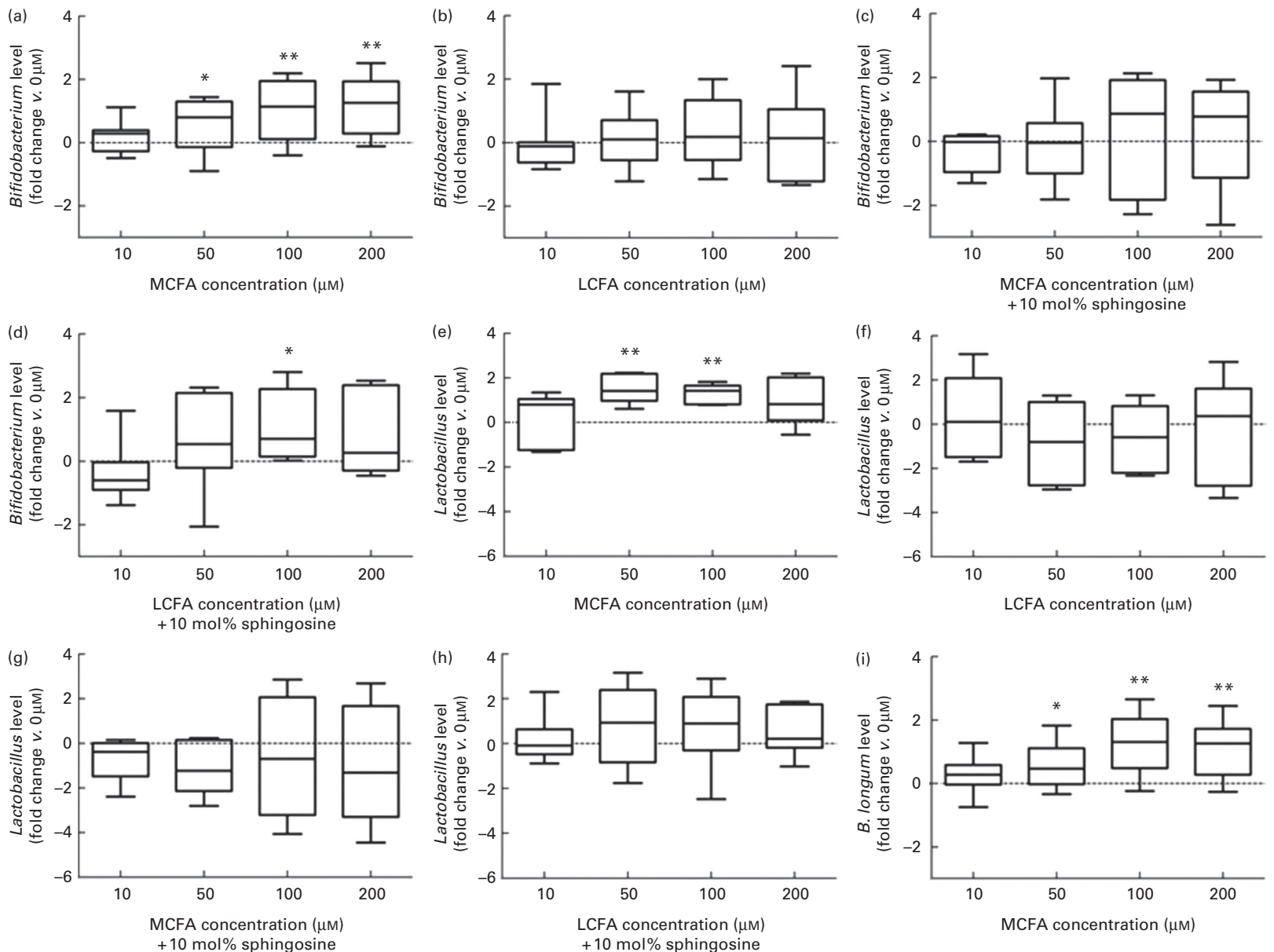

Fig. 2. Quantitative PCR data showing the relative abundance of Bifidobacterium and Lactobacillus as fold difference (log 2 ) compared with the controls after a $24 \mathrm{~h}$ in vitro fermentation. Results are shown for Bifidobacterium after the addition of (a) medium-chained fatty acids (MCFA ( $n$ 9), $50 \mu \mathrm{M}$ : $q=0.039 ; 100 \mu \mathrm{M}$ : $q=0.029 ; 200 \mu \mathrm{M}: q=0.029$ ), (b) long-chained fatty acids (LCFA, $n$ 9), (c) MCFA in combination with $10 \%$ sphingosine, and (d) LCFA in combination with $10 \%$

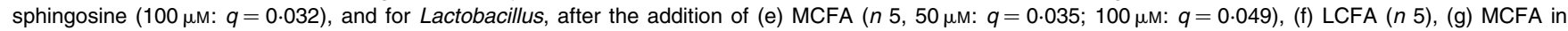
combination with $10 \%$ sphingosine $(n 5)$, and (h) LCFA in combination with $10 \%$ sphingosine $(n 5)$. (i) Correlation between the concentrations of MCFA and the relative abundance of $B$. longum ( $n 9,50 \mu \mathrm{M}: q=0.039 ; 100 \mu \mathrm{M}: q=0.029 ; 200 \mu \mathrm{M}: q=0.029)$. Data are presented as box and whisker plots, with box representing 25-75th percentiles and whisker representing 5-95th percentiles. Value was significantly different from that of the controls $(0 \mu \mathrm{M}):{ }^{\star} P<0.05,{ }^{\star \star} P<0.01$.

correlation test revealed that the higher relative abundance of Bifidobacterium was significantly correlated with the concentrations of MCFA ranging from 0 to $100 \mu \mathrm{M}(P=0.0035$, $R^{2}$ 0.9930; results not shown). The species $B$. adolescentis, B. pseudocatenulatum and B. breve were only found in two to four of the samples. However, the tendency to increasing abundance with increasing concentration was similar for all the tested Bifidobacterium species (results not shown).

Effect of fermentation with lipids on lactic acidproducing bacteria as determined by $16 \mathrm{~S}$ ribosomal RNA gene sequencing. To further characterise the lipid-induced changes in the microbiota, treatments in which changes in Lactobacillus and Bifidobacterium were observed were further sequenced. As the preliminary effects were observed mainly at concentrations of 50 and $100 \mu \mathrm{M}$, only these concentrations (as well as the controls) were included in the sequencing.

Ion Torrent ${ }^{\circledR}$ sequencing generated in total $3 \cdot 2$ million high-quality reads, and the number of reads per sample was $30 \cdot 044-61 \cdot 084$, giving an average of $47 \cdot 155$ reads per sample (median 44.785). On average, more than $99 \%$ of the bacteria were classified at the genus level.

Sequencing revealed a number of changes caused by the addition of MCFA and LCFA + sphingosine at the phylum, family and genus levels. The effects are summarised in Table 2.

Both MCFA and LCFA + sphingosine affected the relative abundance of Actinobacteria (Table 2). Consistent with the qPCR data, the genus Bifidobacterium was observed to be 3 -fold higher after the addition of both types of lipid mixtures at the concentration of $100 \mu \mathrm{M}$. Also concentrations of $50 \mu \mathrm{M}$ tended to increase the relative abundance of this genus (MCFA: $P=0.032, q=0.064 ;$ LCFA + sphingosine: $P=0.035$, $q=0.063)$. Within the Coriobacteriaceae, the genus Eggerthella was approximately 3-fold higher after the addition of MCFA at $50 \mu \mathrm{M}$, whereas this effect was only a tendency after the addition of $100 \mu \mathrm{M}(P=0.045, q=0.082)$.

Within the Proteobacteria, only the addition of LCFA + sphingosine showed an effect. Here, the addition of $100 \mu \mathrm{M}$ significantly decreased the relative abundance of this 
Table 2. Summary of the effects observed by the addition of lipid mixture on the relative abundance of different bacteria at the phylum, family and genus levelsł

\begin{tabular}{|c|c|c|c|c|c|c|c|}
\hline \multirow[b]{2}{*}{ Phylum } & \multirow[b]{2}{*}{ Family } & \multirow[b]{2}{*}{ Genus } & \multirow[b]{2}{*}{$n$} & \multicolumn{2}{|c|}{ MCFA } & \multicolumn{2}{|c|}{ LCFA + sphingosine } \\
\hline & & & & $50 \mu \mathrm{M}$ & $100 \mu \mathrm{M}$ & $50 \mu \mathrm{M}$ & $100 \mu \mathrm{M}$ \\
\hline \multirow[t]{5}{*}{ Actinobacteria } & & & 9 & 2.06 & $2 \cdot 91 * \dagger$ & 3.05 & $3 \cdot 10 * \dagger$ \\
\hline & Bifidobacteriaceae & & 9 & 2.03 & $2.97 * \dagger$ & 3.05 & $3.07 * \dagger$ \\
\hline & & Bifidobacterium & 9 & 1.97 & $2 \cdot 96 * \dagger$ & 3.05 & $3.07 * \dagger$ \\
\hline & Coriobacteriaceae & & 6 & $2.55^{*} \dagger$ & $2 \cdot 23$ & $2 \cdot 89$ & $3 \cdot 29$ \\
\hline & & Eggerthella & 5 & $3 \cdot 18^{*} \dagger$ & 2.48 & $2 \cdot 21$ & 2.09 \\
\hline \multirow[t]{2}{*}{ Proteobacteria } & & & 9 & 0.94 & 0.92 & $0 \cdot 89$ & $0.79 * \dagger$ \\
\hline & Enterobacteriaceae & & 9 & 0.95 & 0.92 & $0.90^{*}$ & $0.82^{*}$ \\
\hline \multirow[t]{8}{*}{ Firmicutes } & & & & & & & \\
\hline & Lactobacillaceae & & 6 & $3 \cdot 77^{*} \dagger$ & $3.54 * \dagger$ & $2 \cdot 58$ & $2 \cdot 64$ \\
\hline & & Lactobacillus & 6 & $3 \cdot 76 * \dagger$ & $3.53 * \dagger$ & 2.58 & $2 \cdot 64$ \\
\hline & Streptococcaceae & & 8 & 1.57 & $2 \cdot 25 * \dagger$ & $1 \cdot 16$ & 1.72 \\
\hline & & Streptococcus & 8 & 1.58 & $2 \cdot 25 * \dagger$ & $1 \cdot 15$ & 1.72 \\
\hline & Lachnospiraceae & & 5 & $0.65^{*} \dagger$ & $1 \cdot 13$ & 0.97 & 1.50 \\
\hline & Erysipelotrichaceae & & 4 & $0.77^{*}$ & 0.99 & $0.60 * \dagger$ & 1.04 \\
\hline & & Clostridium XVIII & 4 & 0.87 & 1.04 & $0.64 * \dagger$ & $1 \cdot 10$ \\
\hline \multirow[t]{3}{*}{ Bacteroidetes } & & & & & & & \\
\hline & Bacteroidaceae & & 6 & 0.75 & $0.67 * \dagger$ & 0.44 & 0.85 \\
\hline & & Bacteroides & 6 & 0.75 & $0.67^{*} \dagger$ & 0.44 & 0.85 \\
\hline
\end{tabular}

MCFA, medium-chained fatty acids; LCFA, long-chained fatty acids.

${ }^{*} P<0.05$.

$\dagger q<0.05$.

$\ddagger$ Green, higher relative abundance compared with the controls; red, lower relative abundance compared with the controls; white, no difference in relative abundance compared with the controls. Colour intensity indicates the relative magnitude of the effect. Effects are showed as fold changes compared with the controls $(0 \mu \mathrm{M})$.

phylum. Additionally, there was a tendency that the family Enterobacteriaceae was significantly decreased at both 50 and $100 \mu \mathrm{M}$.

The relative abundance of Bacteroides was 0.6-fold lower after the addition of MCFA at $100 \mu \mathrm{M}$; however, this effect was not observed at the concentration of $50 \mu \mathrm{M}(P=0.680$, $q=0 \cdot 285)$

In agreement with the qPCR data (Fig. 2), a 3.7-fold higher relative abundance of Lactobacillus was observed after the addition of MCFA at both 50 and $100 \mu \mathrm{m}$. The relative abundance of Streptococcus was significantly higher after the addition of MCFA at $100 \mu \mathrm{m}$. This effect was not observed by the addition of MCFA at $50 \mu \mathrm{M}(P=0 \cdot 207, q=0 \cdot 170)$. Conversely, the relative abundance of the families Lachnospiraceae and Erysipelotrichaceae were significantly lower. Lachnospiraceae was 0.6-fold lower after the addition of MCFA at $50 \mu \mathrm{m}$, and Erysipelotrichaceae was 0.6-fold lower after the addition of LCFA + sphingosine at $50 \mu \mathrm{m}$. However, concentrations at $100 \mu \mathrm{m}$ did not have any effect on either of these families.

The principal component analysis based on bacterial distributions between the genera (Fig. 3) revealed that the addition of both MCFA and LCFA + sphingosine significantly affected the distribution along PC1 in the score plot, where the addition of either lipid mixture shifted the scores for each individual sample at PC1 towards lower values (Fig. 3(a) and (d)). As observed from the loading plots for both MCFA and LCFA + sphingosine (Fig. 3(b) and (d)), this is caused by a shift in the bacterial composition away from Proteobacteria (defined by Escherichia/Shigella and Veillonella) towards lactic acid-producing genera such as Bifidobacterium, Enterococcus, Actinomyces, Lactobacillus and Streptococcus, which all have loading values $\leq-0 \cdot 4$.
Using a Wilcoxon matched-pairs signed-rank test, it was found that addition of MCFA at $100 \mu \mathrm{m}$, as well as of LCFA + sphingosine at both 50 and $100 \mu \mathrm{m}$ significantly lowered the PC1 values compared with the controls (Fig. 3(c) and (f)).

Effect of fermentation without added lipids. Comparison of bacterial communities originating from before (original inoculum) and after (control samples) the $24 \mathrm{~h}$ in vitro fermentation revealed that the biggest change occurring during fermentation without added lipids occurred within the genera Escherichial Shigella and Bifidobacterium. The initial percentage of Escherichia/Shigella in the inoculum was on average 1.07 (SD 1.34 ) $\%$ of the total genera; however, after the $24 \mathrm{~h}$ in vitro fermentation, this level was significantly increased $(P=0.006$; Student's $t$ test) to levels up to $94.7 \%$ (40.7 (sD 32.8)\%) (Fig. 4(a)). In two sample communities, levels remained at approximately $0.1 \%$ both before and after the fermentation.

In contrast, the relative abundance of Bifidobacterium was significantly decreased $(P=0 \cdot 012)$ from more than $55 \%(53 \cdot 1$ (SD 34.2) \%) to less than 33\% (15.4 (sD 12.0)\%) after the $24 \mathrm{~h}$ in vitro fermentation (Fig. 4(b)). The relative abundance of Lactobacillus was only increased in two of the individuals, while the others remained unaffected by the fermentation (Fig. 4(c)). However, Lactobacillus was only present in five of nine individuals.

SCFA analysis. To investigate the functional effects caused by the change in the composition of the microbial communities, the amounts of SCFA in supernatants from the fermentation medium were determined. As the most pronounced effect on bacterial distribution was observed at a concentration of $100 \mu \mathrm{M}$, these samples were selected for the measurements of SCFA. The addition of MCFA (Fig. 5(a)) and MCFA + sphingosine (Fig. 5(b)) did not affect SCFA production. Fermentation with 
R. G. Nejrup et al.
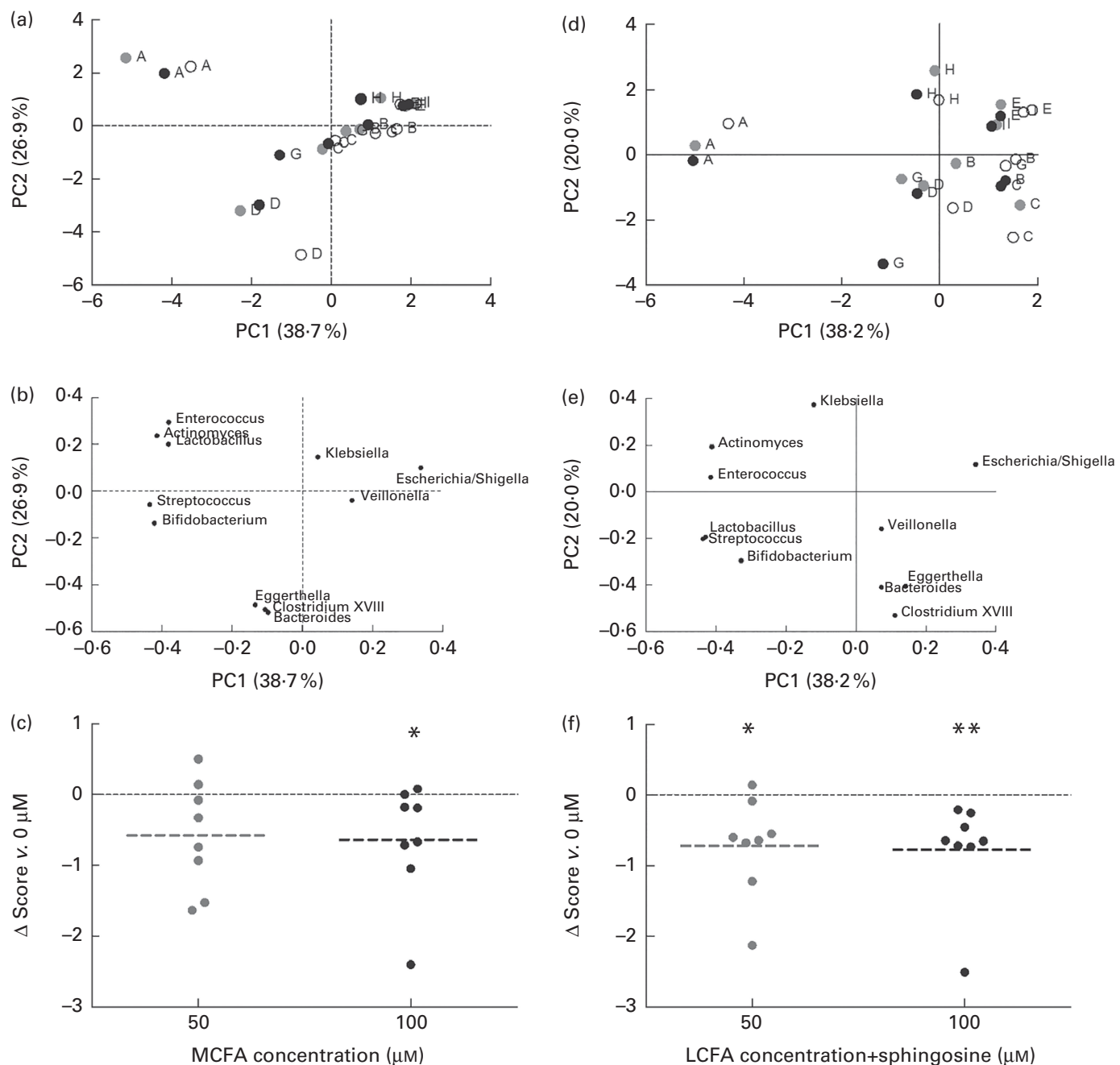

Fig. 3. Principal component $(P C)$ analysis plot showing the effect of $(a-c)$ medium-chained fatty acids (MCFA) and (d-f) long-chained fatty acids (LCFA)+ $10 \mathrm{~mol} \%$ sphingosine on bacterial distribution at the genus level. The colours on score plots $(a, d)$ represent three different concentrations of lipid addition to the fermentation medium $(\mathrm{O}, 0 \mu \mathrm{M} ; \mathrm{O}, 50 \mu \mathrm{M} ; \bullet, 100 \mu \mathrm{M})$. Letters $(\mathrm{A}-\mathrm{J})$ indicate each individual. Loading plots $(\mathrm{b}, \mathrm{e})$ are distributed by PC1 and PC2.. Scores (c and f) are calculated compared with the controls $(0 \mu \mathrm{M})$. Value was significantly different from that of the controls $(0 \mu \mathrm{M}):{ }^{*} P<0.05,{ }^{* \star} P<0.01$.

added LCFA and LCFA + sphingosine resulted in either significantly higher or lower levels of propionic acid, respectively, than measured levels in the controls (Fig. 5(c) and (d)). All the other SCFA remained unchanged.

\section{Discussion}

The fermentation medium used in the present study was an enriched medium containing both simple and complex carbohydrates. Simple carbohydrates are normally absorbed for

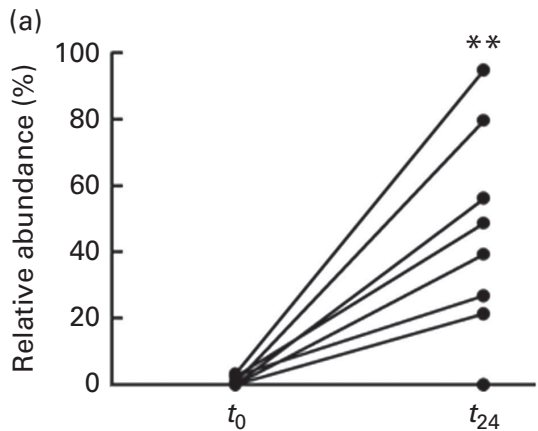

(b)

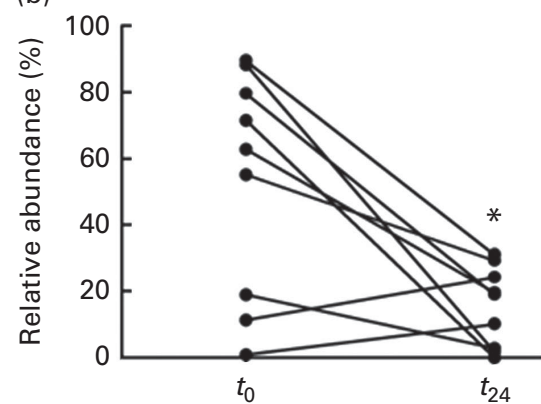

(c)

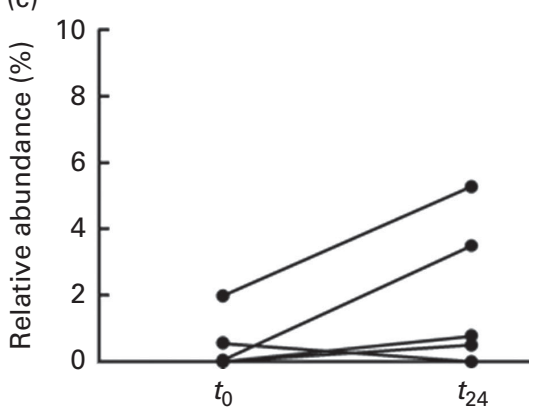

Fig. 4. Effect of the fermentation medium itself shown as the relative abundance of (a) Escherichia/Shigella, (b) Bifidobacterium and (c) Lactobacillus at $t_{0}$ and $t_{24}$, respectively. Each line represents one individual. Value was significantly different from that at time $0:{ }^{*} P<0.05,{ }^{* *} P<0.01$. 
(a)

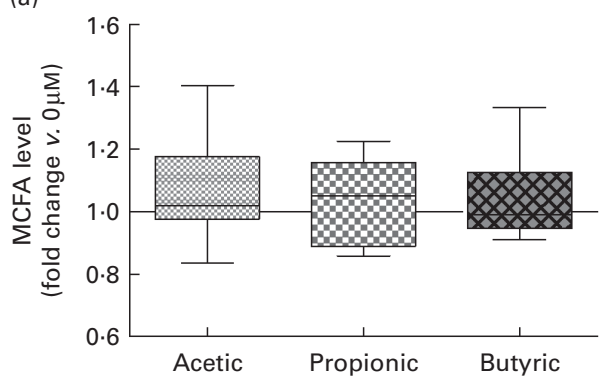

(c)

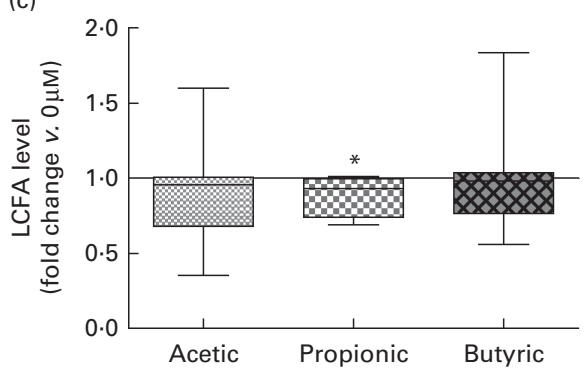

(b)
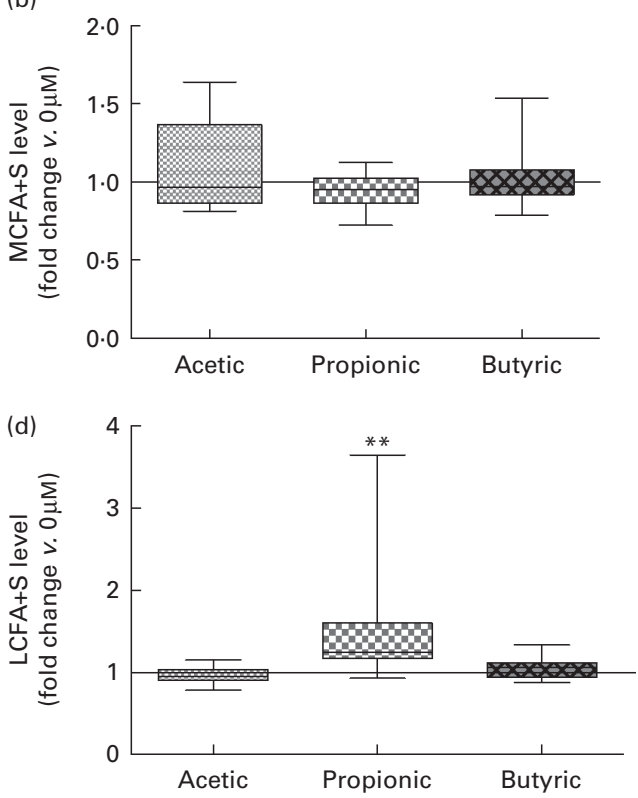

Fig. 5. SCFA levels in supernatants from the fermentation medium containing lipid mixture at a concentration of $100 \mu \mathrm{M}$. Levels are shown as fold changes (log 2 compared with the controls $(0 \mu \mathrm{M})$. Results are shown for the addition of (a) medium-chained fatty acids (MCFA) (b) MCFA +10 mol\% sphingosine (c) longchained fatty acids (LCFA) and (d) LCFA $+10 \mathrm{~mol} \%$ sphingosine. Data are presented as box and whisker plots, with box representing $25-75$ th percentiles and whisker representing $5-95$ th percentiles. Value was significantly different from that of the controls $(0 \mu \mathrm{M})$ : ${ }^{\star} P<0.05,{ }^{\star \star} P<0.01$.

energy extraction in the duodenum and upper ileum, and thus less likely to reach the lower ileum and colon where bacterial fermentation takes place. However, as the aim of the present study was to investigate the effect of lipid mixture addition, and not the bacterial ability to grow on FA as their sole carbohydrate source, it was essential to design the fermentation medium not to be limiting the availability of bacterial carbohydrates.

Under these carbohydrate-rich conditions, we investigated the effect of lipid hydrolysis products from milk fat on the bacterial composition of the gut microbiota isolated from nine healthy infants. Sequencing and qPCR of $16 \mathrm{~S}$ rRNA gene regions was applied to examine the composition of bacteria in communities derived from infant faeces after a $24 \mathrm{~h}$ anaerobic in vitro fermentation.

Classification at the phylum, family and genus levels revealed, as expected, a much less complex microbiota in infants than normally seen in adults ${ }^{(42)}$, probably due to consumption of a less complex diet during the early part of life. The composition was found to vary greatly between infants, confirming previous findings ${ }^{(43)}$. Despite the vast compositional variation between samples, the microbial communities responded almost uniformly to the different lipid additions, which showed highly specific effects on the growth of certain bacterial genera.

Overall, four different combinations of lipids were tested for their effect on bacterial abundance; however, in general, the MCFA and LCFA + sphingosine had the greatest influence.

Both MCFA and LCFA + sphingosine caused a significantly higher relative abundance of Bifidobacterium, including $B$. breve, B. adolescentis and B. pseudocatenulatum compared with the controls. Apart from this, the addition of MCFA resulted in a higher relative abundance of Lactobacillus. In agreement with the present results, a study in broilers has shown that a diet rich in MCFA promoted specific Lactobacillus species, while species belonging to the families Micrococcaceae and Enterococcaceae were suppressed ${ }^{(44)}$. Another study in piglets has demonstrated an increase in the abundance of L. johnsonii as a result of MCFA-enriched diet $^{(34)}$. Thus, there is accumulating evidence that MCFA can have a specific stimulatory effect on Lactobacillus. The sequencing data also showed that both MCFA and LCFA + sphingosine caused a general shift of the microbiota, reducing the abundance of Proteobacteria and increasing the abundance of lactic acid-producing bacteria (Fig. 3), which must be considered as a positive effect in the infant gut ${ }^{(45)}$. This may be caused by a higher sensitivity of the Gram-negative Proteobacteria to FA, giving a competitive advantage to acid-tolerant lactic acid producers. The relative increase in the amount of Bifidobacterium in MCFA- and LCFA + sphingosine-supplemented fermentations, compared with the controls, seems to be explained by a reduced abundance of these bacteria in the controls, rather than by an actual increase in the lipid-supplemented fermentations. This supports the interpretation that the effect of the lipids on bifidobacteria may rely primarily on the competitive advantage caused by an antagonistic effect of the lipids on competing species such as Proteobacteria.

The observed changes in the microbiota composition, with a relative increased concentration of SCFA producers such as Bifidobacterium and Lactobacillus, could imply a higher production of SCFA after the addition of MCFA and LCFA + sphingosine. Therefore, we analysed the concentration of SCFA in the fermentation fluid. Despite the relatively 
pronounced changes in the microbiota, there were no changes in SCFA content or composition after the addition of MCFA and only an increase in propionic acid was observed after the addition of LCFA + sphingosine. Although superficially surprising, bacterial production of SCFA is highly dependent on their access to carbon sources, and when carbohydrates are not limited, as in the present study, it is known that, for example, Bifidobacterium species produce more lactate as metabolic end products ${ }^{(46)}$. Lactate is then rapidly consumed by other species in the community. Hence, the fact that we have fermented the microbiota samples in carbohydrate-excess conditions might explain why the observed relative increase in the populations of Bifidobacterium and Lactobacillus does not lead to concomitant increased SCFA production.

MCFA and sphingosine have previously been studied for their antibacterial effects against pathogenic bacteria on human $\operatorname{skin}^{(22,24)}$.

It is not plausible that the stimulated lactic acid-producing Gram-positive bacteria are utilising FA as carbon source. As the fermentation medium contains both simple and complex carbohydrates, these carbon sources would have to be degraded before bacterial metabolism switches to lipid metabolism $^{(47)}$. However, as several studies have demonstrated that unsaturated FA and oleic acid, in particular, stimulates the growth of specific Lactobacillus species ${ }^{(26)}$, we had expected that the growth of Lactobacillus would be stimulated by the addition of LCFA, but this was not observed. One possible explanation may be that other bacteria, such as Escherichial Shigella, which were observed to be favoured by the in vitro conditions, may overgrow the low-abundant Lactobacillus in our model system. The relative stimulation of Lactobacillus in the presence of MCFA would then be explained by either a stronger inhibition of Escherichia/Shigella under these conditions, or a specific positive effect on Lactobacillus growth by MCFA, or a combination of both.

The lipid mixtures in the present study were applied in concentrations ranging from 10 to $200 \mu \mathrm{M}$, and significant effects were observed for concentrations $\geq 50 \mu \mathrm{m}$. It has been reported that the concentration of caprylic acid $(8: 0)$ and capric acid $(10: 0)$ in the caecum and distal jejunum of piglets is about $100 \mu \mathrm{mol} / \mathrm{kg}$ lumen content and even higher in the proximal and mid-jejunum ${ }^{(34)}$. Hence, the applied concentrations are within a physiological range. In general, the effects observed by the addition of lipid mixtures at 100 and $200 \mu \mathrm{M}$, respectively, were similar, indicating that bacteria tolerating a concentration of $100 \mu \mathrm{m}$ also tolerate higher concentrations, and that the maximal inhibitory effect on competing bacteria is thus reached at $100 \mu \mathrm{m}$.

Breast milk is unique in its relatively high content of MCFA. This is considered mainly to be due to the fact that MCFA are easier and rapidly absorbed and oxidised for energy extraction than LCFA, since they are primarily absorbed via the portal vein directly to the liver and rapidly transferred to the mitochondrial matrix independent of the carnitine shuttle system $^{(48)}$. However, the present results suggest that these FA also play another important role in infancy, namely the modulation of the gut microbiota. During this very early period of life, where energy extraction and maturation of the immune system is essential for optimal growth and development, an increase in the growth of lactic acid-producing bacteria (i.e. Bifidobacterium and Lactobacillus) and the reduced abundance of Proteobacteria in the establishing gut microbiota may very well be beneficial.

The present study contributes to the development of new nutritional strategies intended to modulate the microbiota in infants, which may support a healthy development of the metabolic and immune system.

\section{Acknowledgements}

The authors thank all voluntary mothers for accepting their infants' participation in the study as well as the funding bodies (see below). Additionally, the laboratory technicians Bodil Madsen, Kate Vina Vibefeldt and Jannie Felskov Agersten are thanked for their excellent technical support.

The authors thank the Danish Dairy Research Foundation, Arla Food Ingredients and Technical University of Denmark for funding the present study. Additionally, scientific support was obtained through collaboration with the Gut, Grain \& Greens (3G) Center, supported by the Danish Council for Strategic Research (grant no. 11-116163).

The present study was partly funded by Arla Foods Ingredients. The funder had no influence on the development of the research, experimental design, data interpretation or the content of the manuscript.

The contributions of the authors are as follows: R. G. N. participated in the planning of the study, and performed the main part of the experimental work, data analysis and interpretation, and writing the first draft of the manuscript; M. I. B. was involved in the sequencing and qPCR analysis, interpreting the data from the analysis and critically read the manuscript; L. K. V. participated in the design of the fermentation set-up and critically read the manuscript; C. H. conducted the SCFA analysis and critically read the manuscript; T. R. L. conceived the project idea with L. I. H., planned and designed the study in collaboration with R. G. N. and L. I. H., interpreted the data and co-authored the final manuscript; L. I. H. conceived the initial project idea with T. R. L., planned and designed the study with R. G. N. and T. R. L., performed the data analysis together with R. G. N., interpreted data and co-authored the final manuscript.

There are no conflicts of interest.

\section{References}

1. Scholtens PA, Oozeer R, Martin R, et al. (2012) The early settlers: intestinal microbiology in early life. Annu Rev Food Sci Technol 3, 425-447.

2. Solis Get al.de los Reyes-Gavilan CG \& Fernandez N (2010) Establishment and development of lactic acid bacteria and bifidobacteria microbiota in breast-milk and the infant gut. Anaerobe 16, 307-310.

3. Mackie RI, Sghir A \& Gaskins HR (1999) Developmental microbial ecology of the neonatal gastrointestinal tract. Am J Clin Nutr 69, 1035s-1045s. 
4. Fanaro S, Chierici R, Guerrini P, et al. (2003) Intestinal microflora in early infancy: composition and development. Acta Paediatr Suppl 91, 48-55.

5. Orrhage K \& Nord CE (1999) Factors controlling the bacterial colonization of the intestine in breastfed infants. Acta Paediatr Suppl 88, 47-57.

6. Rerksuppaphol S \& Rerksuppaphol L (2010) Lactobacillus acidophilus and Bifidobacterium bifidum stored at ambient temperature are effective in the treatment of acute diarrhoea. Ann Trop Paediatr 30, 299-304.

7. Braga TD, da Silva GA, de Lira PI, et al. (2011) Efficacy of Bifidobacterium breve and Lactobacillus casei oral supplementation on necrotizing enterocolitis in very-lowbirth-weight preterm infants: a double-blind, randomized, controlled trial. Am J Clin Nutr 93, 81-86.

8. Turroni F, Peano C, Pass DA, et al. (2012) Diversity of bifidobacteria within the infant gut microbiota. PLOS ONE 7, e36957.

9. Macfarlane GT \& Macfarlane LE (2009) Acquisition, evolution and maintenance of the normal gut microbiota. Dig Dis 27, Suppl. 1, 90-98.

10. Lindquist $S$ \& Hernell $O$ (2010) Lipid digestion and absorption in early life: an update. Curr Opin Clin Nutr Metab Care 13, 314-320.

11. Andersson EL, Hernell O, Blackberg L, et al. (2011) BSSL and PLRP2: key enzymes for lipid digestion in the newborn examined using the Caco-2 cell line. J Lipid Res 52, 1949-1956.

12. Francois CA, Connor SL, Wander RC, et al. (1998) Acute effects of dietary fatty acids on the fatty acids of human milk. Am J Clin Nutr 67, 301-308.

13. Gibson RA \& Kneebone GM (1981) Fatty acid composition of human colostrum and mature breast milk. Am J Clin Nutr 34, $252-257$.

14. Berenhauser AC, Pinheiro do Prado AC, da Silva RC, et al. (2012) Fatty acid composition in preterm and term breast milk. Int J Food Sci Nutr 63, 318-325.

15. Lubetzky R, Zaidenberg-Israeli G, Mimouni FB, et al. (2012) Human milk fatty acids profile changes during prolonged lactation: a cross-sectional study. Isr Med Assoc J 14, 7-10.

16. Jensen RG (1999) Lipids in human milk. Lipids 34, 1243-1271.

17. Yuhas R, Pramuk K \& Lien EL (2006) Human milk fatty acid composition from nine countries varies most in DHA. Lipids 41, 851-858.

18. Neville MC \& Picciano MF (1997) Regulation of milk lipid secretion and composition. Annu Rev Nutr 17, 159-183.

19. Lopez C \& Menard O (2011) Human milk fat globules: polar lipid composition and in situ structural investigations revealing the heterogeneous distribution of proteins and the lateral segregation of sphingomyelin in the biological membrane. Colloids and Surf B Biointerfaces 83, 29-41.

20. Zou XQ, Guo Z, Huang JH, et al. (2012) Human milk fat globules from different stages of lactation: a lipid composition analysis and microstructure characterization. J Agric Food Chem 60, 7158-7167.

21. Nilsson A \& Duan RD (2006) Absorption and lipoprotein transport of sphingomyelin. J Lipid Res 47, 154-171.

22. Sprong RC, Hulstein MF \& Van der Meer R (2001) Bactericidal activities of milk lipids. Antimicrob Agents Chemother 45, 1298-1301.

23. Drake DR, Brogden KA, Dawson DV, et al. (2008) Thematic review series: skin lipids. Antimicrobial lipids at the skin surface. J Lipid Res 49, 4-11.

24. Fischer CL, Drake DR, Dawson DV, et al. (2012) Antibacterial activity of sphingoid bases and fatty acids against gram-positive and gram-negative bacteria. Antimicrob Agents Chemother 56, 1157-1161.

25. Sprong RC \& Hulstein MFE (2002) van der Meer R Bovine milk fat components inhibit food-borne pathogens. Int Dairy J 12, 209-215.

26. Williams VR \& Fieger EA (1946) Oleic acid as a growth stimulant for Lactobacillus casei. J Biol Chem 166, 335-343.

27. Partanen L, Marttinen N \& Alatossava T (2001) Fats and fatty acids as growth factors for Lactobacillus delbrueckii. Syst Appl Microbiol 24, 500-506.

28. Corcoran BM, Stanton C, Fitzgerald GF, et al. (2007) Growth of probiotic lactobacilli in the presence of oleic acid enhances subsequent survival in gastric juice. Microbiology 153, 291-299.

29. Muller JA, Ross RP, Sybesma WF, et al. (2011) Modification of the technical properties of Lactobacillus johnsonii NCC 533 by supplementing the growth medium with unsaturated fatty acids. Appl Environ Microbiol 77, 6889-6898.

30. Abrahamse CE \& Bartowsky EJ (2012) Timing of malolactic fermentation inoculation in Shiraz grape must and wine: influence on chemical composition. World J Microbiol Biotechnol 28, 255-265.

31. Armand M, Hamosh M, Mehta NR, et al. (1996) Effect of human milk or formula on gastric function and fat digestion in the premature infant. Pediatr Res 40, 429-437.

32. Roman C, Carriere F, Villeneuve P, et al. (2007) Quantitative and qualitative study of gastric lipolysis in premature infants: do MCT-enriched infant formulas improve fat digestion? Pediatr Res 61, 83-88.

33. Chappell JE, Clandinin MT, Kearney-Volpe C, et al. (1986) Fatty acid balance studies in premature infants fed human milk or formula: effect of calcium supplementation. J Pediatr 108, 439-447.

34. Zentek J, Buchheit-Renko S, Manner K, et al. (2012) Intestinal concentrations of free and encapsulated dietary mediumchain fatty acids and effects on gastric microbial ecology and bacterial metabolic products in the digestive tract of piglets. Arch Anim Nutr 66, 14-26.

35. Azad MB, Konya T, Maughan H, et al. (2013) Gut microbiota of healthy Canadian infants: profiles by mode of delivery and infant diet at 4 months. CMAJ 185, 385-394.

36. Sela DA \& Mills DA (2010) Nursing our microbiota: molecular linkages between bifidobacteria and milk oligosaccharides. Trends Microbiol 18, 298-307.

37. Rycroft CE, Jones MR, Gibson GR, et al. (2001) A comparative in vitro evaluation of the fermentation properties of prebiotic oligosaccharides. J Appl Microbiol 91, 878-887.

38. Bergstrom A, Licht TR, Wilcks A, et al. (2012) Introducing GUt low-density array (GULDA): a validated approach for qPCR-based intestinal microbial community analysis. FEMS Microbiol Lett 337, 38-47.

39. Christensen EG, Licht TR, Leser TD, et al. (2014) Dietary Xylo-oligosaccharide stimulates intestinal bifidobacteria and lactobacilli but has limited effect on intestinal integrity in rats. BMC Res Notes 7, 660.

40. Wang Q, Garrity GM, Tiedje JM, et al. (2007) Naive Bayesian classifier for rapid assignment of rRNA sequences into the new bacterial taxonomy. Appl Environ Microbiol 73, 5261-5267.

41. Zhao GH, Nyman M \& Jonsson JA (2006) Rapid determination of short-chain fatty acids in colonic contents and faeces of humans and rats by acidified water-extraction and direct-injection gas chromatography. Biomed Chroma$\operatorname{tog} r$ 20, 674-682.

42. Qin J, Li R, Raes J, et al. (2010) A human gut microbial gene catalogue established by metagenomic sequencing. Nature 464, 59-65. 
43. Palmer C, Bik EM, DiGiulio DB, et al. (2007) Development of the human infant intestinal microbiota. PLoS Biol 5, e177.

44. van der Hoeven-Hangoor E, van der Vossen JM, Schuren FH, et al. (2013) Ileal microbiota composition of broilers fed various commercial diet compositions. Poult Sci 92, 2713-2723.

45. Shokryazdan P, Sieo CC, Kalavathy R, et al. (2014) Probiotic potential of Lactobacillus strains with antimicrobial activity against some human pathogenic strains. Biomed Res Int 2014, 927268.

46. Macfarlane S \& Macfarlane GT (2003) Regulation of shortchain fatty acid production. Proc Nutr Soc 62, 67-72.

47. Strable MS \& Ntambi JM (2010) Genetic control of de novo lipogenesis: role in diet-induced obesity. Crit Rev Biochem Mol Biol 45, 199-214.

48. Zentek J, Buchheit-Renko S, Ferrara F, et al. (2011) Nutritional and physiological role of medium-chain triglycerides and medium-chain fatty acids in piglets. Anim Health Res Rev 12, 83-93.

49. Walter J, Tannock GW, Tilsala-Timisjarvi A, et al. (2000) Detection and identification of gastrointestinal Lactobacillus species by using denaturing gradient gel electrophoresis and species-specific PCR primers. Appl Environ Microbiol 66, 297-303.

50. Delroisse JM, Boulvin AL, Parmentier I, et al. (2008) Quantification of Bifidobacterium spp. and Lactobacillus spp. in rat fecal samples by real-time PCR. Microbiol Res 163, 663-670.

51. Matsuki T, Watanabe K, Tanaka R, et al. (1998) Rapid identification of human intestinal bifidobacteria by $16 \mathrm{~S}$ rRNA-targeted species- and group-specific primers. FEMS Microbiol Lett 167, 113-121.

52. Rinne MM, Gueimonde M, Kalliomaki M, et al. (2005) Similar bifidogenic effects of prebiotic-supplemented partially hydrolyzed infant formula and breastfeeding on infant gut microbiota. FEMS Immunol Med Microbiol 43, 59-65.

53. Walter J, Hertel C, Tannock GW, et al. (2001) Detection of Lactobacillus, Pediococcus, Leuconostoc, and Weissella species in human feces by using group-specific PCR primers and denaturing gradient gel electrophoresis. Appl Environ Microbiol 67, 2578-2585.

54. Heilig HG, Zoetendal EG, Vaughan EE, et al. (2002) Molecular diversity of Lactobacillus spp. and other lactic acid bacteria in the human intestine as determined by specific amplification of $16 \mathrm{~S}$ ribosomal DNA. Appl Environ Microbiol 68, $114-123$.

55. Firmesse O, Mogenet A, Bresson JL, et al. (2008) Lactobacillus rhamnosus R11 consumed in a food supplement survived human digestive transit without modifying microbiota equilibrium as assessed by real-time polymerase chain reaction. J Mol Microbiol Biotechnol 14, 90-99. 